\title{
GNSS processing at CODE: status report
}

\author{
Rolf Dach • Elmar Brockmann - Stefan Schaer • \\ Gerhard Beutler • Michael Meindl • Lars Prange • \\ Heike Bock · Adrian Jäggi · Luca Ostini
}

Received: 14 May 2008 / Accepted: 14 October 2008

(C) Springer-Verlag 2008

\begin{abstract}
Since May 2003, the Center for Orbit Determination in Europe (CODE), one of the analysis centers of the International GNSS Service, has generated GPS and GLONASS products in a rigorous combined multi-system processing scheme, which promises the best possible consistency of the orbits of both systems. The resulting products, in particular the satellite orbits and clocks, are easily accessible by the user community. In the first part of this article, we focus on the generation of the combined global products at CODE, where we put emphasis not only on accuracy, but also on completeness. We study the impact of GLONASS on the CODE products, and the benefit of using them. Last, but not least, we introduce AGNES (Automated GNSS Network for Switzerland), a regional tracking network of small extensions (roughly $400 \mathrm{~km}$ East-West, 200 km North-South), which consequently tracks all GNSS satellites and analyzes their measurements using the CODE products.
\end{abstract}

Keywords Orbit determination - GNSS processing · GPS . GLONASS

\section{Introduction}

The Center for Orbit Determination in Europe (CODE) is a joint venture of the Astronomical Institute of the University of Bern (AIUB, Switzerland), the Swiss Federal Office

R. Dach $(\bowtie) \cdot$ G. Beutler · M. Meindl · L. Prange · H. Bock ·

A. Jäggi · L. Ostini

Astronomical Institute of the University of Bern, Sidlerstrasse 5, 3012 Bern, Switzerland

e-mail: rolf.dach@aiub.unibe.ch

E. Brockmann $\cdot$ S. Schaer

Swiss Federal Office of Topography (swisstopo),

Seftigenstrasse 264, 3084 Wabern, Switzerland of Topography (swisstopo, Wabern, Switzerland), and the German Federal Office for Cartography and Geodesy (BKG, Frankfurt a. Main, Germany).

CODE started operations on 21 June 1992 and has generated an uninterrupted series of products since that time. All computations are performed at the AIUB using the Bernese GPS Software in its current development version (Dach et al. 2007b). As each of the International GNSS Service (IGS) Analysis Center, CODE aimes to provide the "best possible" products also considering the aspects of the demanding user community of Earth sciences and survey institutions. The attribute "best possible" is interpreted by CODE as follows:

- First, CODE products shall be "state of the art" from the point of view of the products' accuracy. The IGS Analysis Center Coordinators' reports since 1994 underline that, in the average over the time span 1994-2008, CODE was successful in this respect.

- Second, CODE products shall be complete to the extent possible. This implies in particular the inclusion of satellites, which are set unhealthy by the operators (including even GPS satellites during repositioning events, since December 2003).

- Third, completeness, in the CODE understanding, also means the inclusion of ephemerides for all GNSS (Global Navigation Satellite Systems) for which the observations are openly available.

In this article, we focus on the second and the third of the above attributes. This paper should be viewed as an intermediary report covering activities and developments initiated about 10 years ago and which will eventually (in $5-10$ years) lead to a CODE processing scheme including all GNSS of interest to the demanding GNSS user community. The 
decision to proceed in this direction is supported by all partners of CODE.

CODE gained first experience in processing GLONASS data during the IGEX campaign (Ineichen et al. 2003). The processing scheme was a two-step approach, where products like station coordinates, Earth orientation parameters and troposphere parameters were determined by GPS and kept fixed in the second step, where GLONASS was added to the analysis.

In May 2003 CODE started to include the observations of the GLONASS satellites into a fully combined multi-GNSS processing scheme (Schaer et al. 2005) on the level of GPS and GLONASS observations. Due to this strategy all observations may contribute to the generation of the aforementioned products. The rigorous scheme is applied to all product lines of the IGS (final, rapid, and ultra-rapid). Therefore, all CODE-products (apart from the clock corrections) are now multi-system products.

The most important gain of this consequent multi-GNSS processing scheme is the availability of GPS and GLONASS satellites in the same reference frame. It is therefore left to the discretion of the user to select the subset of satellites to be used in a particular analysis. The inclusion of GLONASS into the CODE routine processing is not a trivial step. First we had to make sure that system-specific biases do not contaminate the traditional products. We are now looking back 5 years in our combined GPS/GLONASS products.

The availability of combined GPS/GLONASS products becomes increasingly more important due to the increasing number of network operators enhancing their permanent networks with GPS/GLONASS combined receivers and antennas. The Swiss Permanent Network AGNES is such an example.

In this paper, we first describe the procedure of our multiGNSS processing and assess the accuracy of the new and traditional products. In addition, we compare the characteristics of both GNSS and study the use and impact of the combined products.

\section{GNSS orbit determination}

\subsection{The GNSS subnetwork of the IGS}

The number and distribution of IGS sites providing GPS and GLONASS data has significantly increased and improved since CODE started its combined analysis in Spring 2003. Figure 1 shows the number of stations in the IGS network providing GLONASS measurements. The number of GLONASS capable sites grew from about $20-30$ by the end of the year 2003. The number of stations remained stable for a long time. With the availability of a new generation of combined GPS/GLONASS receivers, produced by several

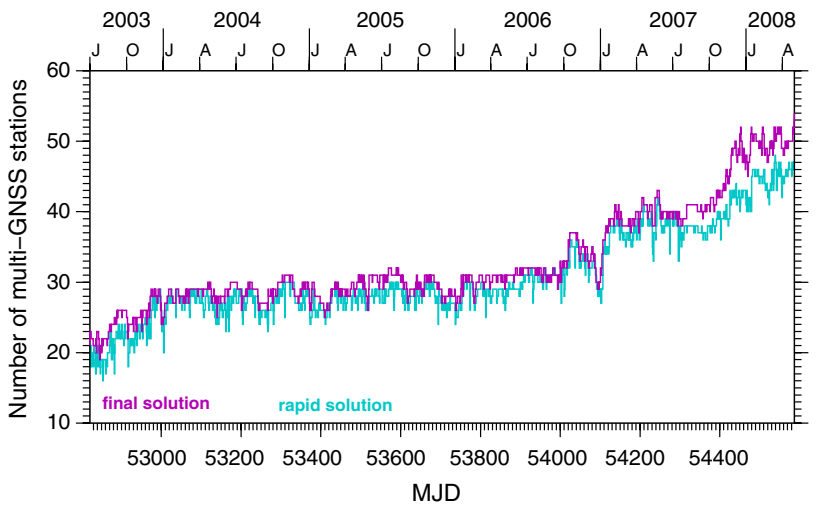

Fig. 1 Number of sites in the IGS network providing GLONASS data, which were used for orbit determination in the CODE rapid (cyan line) and final (magenta line) solution

well-known receiver manufacturers in 2006/2007 the number of GLONASS tracking stations in the IGS network increased steadily and still increases today. The CODE final orbits for the GLONASS satellites are now based on data from 50 tracking stations in the IGS network. For the ultra-rapid solution GLONASS tracking data from about 25 IGS stations are used (the number is limited by the availability of the data). For orbit determination, good global distribution of observing sites is at least as important as their number.

In the summer of 2003, the global coverage of IGS stations tracking GLONASS satellites was highly heterogeneous. Most of the 20 stations with the GLONASS tracking capability were located in Europe (see Fig. 2a). The 30 stations network available in the time interval 2003-2006 is, in essence, that shown in Fig. 2b. Figure 2c shows the current network (early 2008). The relation between GPS-only (green dots) and combined GPS/GLONASS (red stars) receivers is now balanced in all regions-except in the American continent, where GPS-only receivers still dominate. In summary, however, we may state that today orbit determination for the GLONASS satellites may be truly based on a global tracking network of geodetic receivers. This significant improvement is due to the efforts of many IGS station managers and their institutions. We acknowledge this significant contribution.

The number of active GLONASS satellites also grew considerably since 2003. Unfortunately, a large number of receivers were unable to track satellites flagged as "unusable", which reduced the number of receivers tracking these satellites. In 2007, GLONASS moved the frequency range of the system to a new frequency band (announced as a system update already in 2002). The frequencies of the 24 GLONASS satellites of the nominal constellation are no longer computed by the frequency numbers +1 to +12 , but by -7 to +6 . When the first satellites with the frequency number $\leq 0$ became active, several firmware upgrades were necessary to enable the receivers to provide data from these satellites. This (avoidable) receiver problem is responsible 


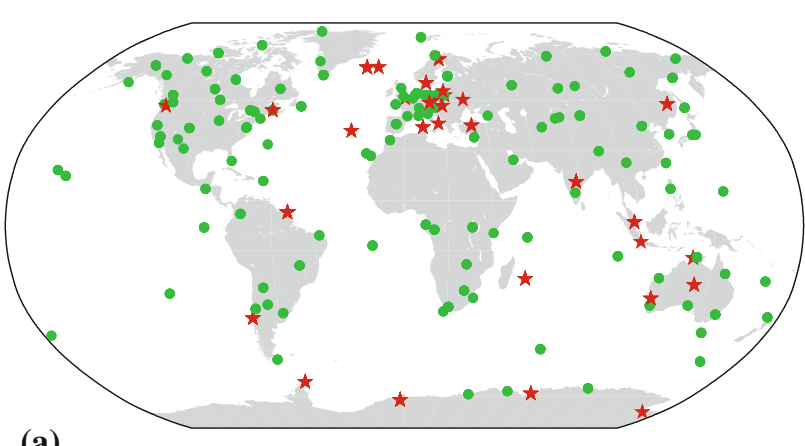

(a)

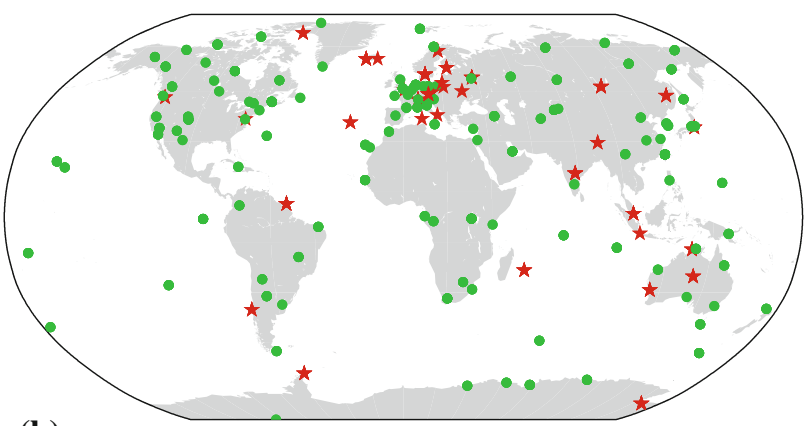

(b)

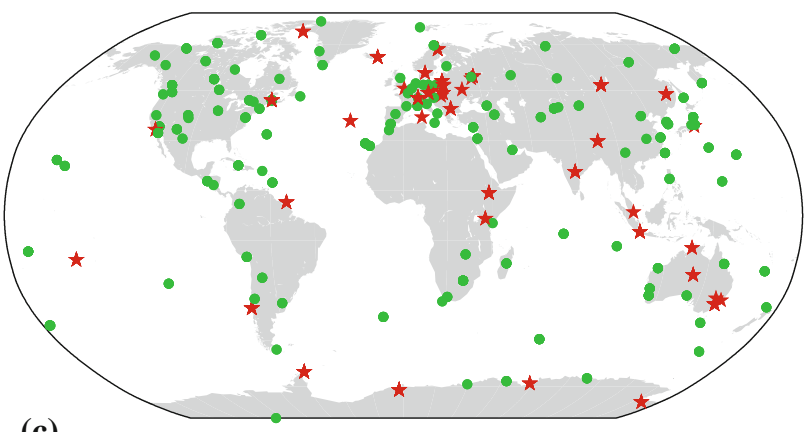

(c)

Fig. 2 Geographical distribution of multi-system GNSS receivers (red stars) and GPS-only receivers (green dots) that are used for the CODE final processing. a GNSS subnetwork of the IGS in July 2003 (day of year 182). b GNSS subnetwork of the IGS in March 2005 (day of year 075). c GNSS subnetwork of the IGS in April 2008 (day of year 110)

that the orbits for some GLONASS satellites had to be computed using the data of only two or three stations for some time periods. For these periods, long arcs of several days were required to guarantee at least a moderate orbit accuracy.

\subsection{Radiation pressure modelling}

The satellites of all GNSS are orbiting the Earth at rather high altitudes (20,000-25,000 km above the Earth's surface). Atmospheric drag may thus be neglected and the Earth's gravity field may be assumed as perfectly known — perhaps with the exception of few low-degree and low-order resonance terms (with Earth rotation).
Radiation pressure is the clearly domination non-gravitational force influencing the GNSS satellites. Unfortunately this force is difficult to take into account in the orbit determination process. One has to make the distinction between the direct radiation pressure (caused by the direct solar radiation) and the albedo radiation pressure, caused by the sunlight reflected from the Earth's surface, atmosphere, or oceans. As the surface properties (specular and diffuse reflection, absorption) of the satellite and its attitude (orientation of the satellite-fixed coordinate system in the inertial space) are not known with sufficient accuracy, empirical parameters have to be solved for in the orbit determination process. If the orientation were known perfectly, it may be sufficient to solve only for a scale factor of a high-quality a priori model. This approach proved to be inadequate for precise orbit determination for GNSS orbits aiming at precision on centimeter level.

The orbit modelling problem may be dealt with in several ways. For example, one may introduce and solve for stochastic accelerations in the satellites' equations of motionan avenue followed by the JPL Analysis Center and centers using its software. At CODE the problem is addressed in a different way by the introduction of an empirical force (acceleration) model. These models are in essence based on the theory developed in Beutler et al. (1994).

The empirical model was modified and generalized by Springer et al. (1999) to serve as an a priori model. Since 1998, CODE has used this empirical radiation pressure model containing a set of parameters for each individual satellite or for groups of satellites of a specific type. The model contains, in particular, constant, once-per-revolution, twice-perrevolution terms, etc., in the sun-satellite direction (D), along the solar panels axes (Y), and (X), the direction perpendicular to (D) and (Y). New coefficient sets for this model have been derived recently from the final CODE orbits of the years 2000-2006 (the preceding set of coefficient was generated in 1998). The October 2006 set contains the coefficients for all GPS satellites (including those launched after 1998 for a first time).

The empirical model was, in essence, fine-tuned using the GPS satellites of all types. Given that very little information was available concerning the GLONASS spacecrafts and attitude control, it seemed logical to use the empirical model from Springer et al. (1999) for GLONASS, as well. The corresponding coefficients were derived from data gathered from 2003 through 2007, for which the GLONASS satellites were included into the CODE analysis.

The quality of the new coefficient was assessed by an orbit prediction over 2 weeks. The prediction is based on 3 days of observations (from the regular processing at CODE), where each orbit was parameterized by six initial osculating elements and two constant direct radiation pressure components in (D) and (Y). All the other components were taken over from the October 2006 a priori model. The predicted 


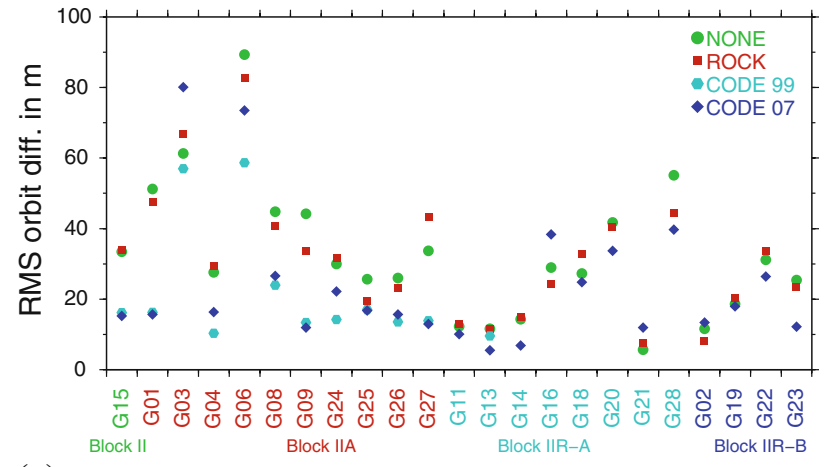

(a)

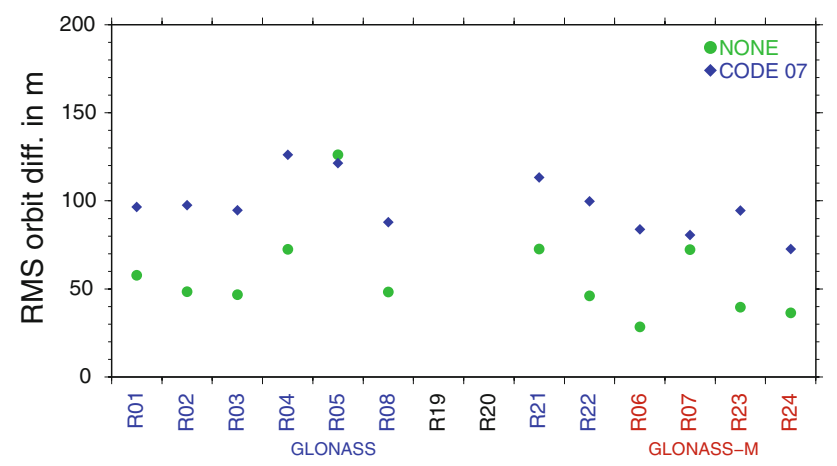

(b)

Fig. 3 RMS differences between estimated orbits ("ground truth") and predicted orbits based on different radiation pressure models. The orbit predictions time span was 2 weeks. Eclipsing satellites are excluded from the differences. a GPS satellites. b GLONASS satellites

positions of the 15th-day are compared to the positions of the final orbit of the same day, where the RMS value for each satellite results from the differences "predicted-final" orbit positions. This prediction procedure was shifted day-by-day over the time interval of 3 months of the year 2005 .

The mean values of all satellite-specific RMS errors for each satellite are provided in Fig. 3. The four series of values shown differ only in the a priori radiation pressure model used: One series of values was generated without an a priori model, the second using the ROCK models provided by Fliegel and Gallini (1996), the third using the old set of parameters as published by Springer et al. (1999), and the fourth using the 2006 set of parameters.

From Fig. 3, we conclude that the new set of coefficients for the CODE model is of comparable quality to the old one. The CODE models are superior in quality to the ROCK models-this is in particular true for the Block IIA satellites. For GLONASS it is obviously best not to use an a priori model.

\subsection{GLONASS orbit determination}

The number of GNSS satellites contained in the CODE final solution is shown in Fig. 4. The green curve shows the number

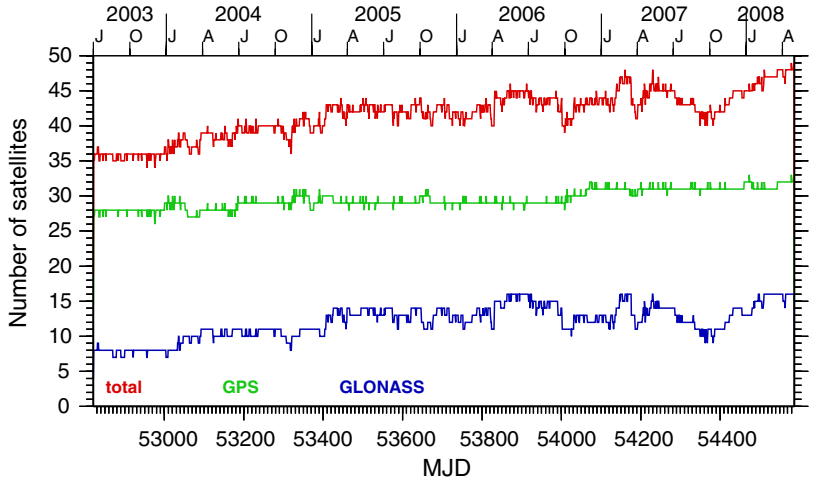

Fig. 4 Number of satellites included in the CODE final orbit product since 2004

of available GPS satellites, which is quite stable around 30 since the year 2000. The number of GLONASS satellites tracked by a sufficient number of sites of the IGS network to allow for precise orbit determination is represented by the blue curve in Fig. 4. This number shows much larger variations than the corresponding GPS curve.

This variation is explained by two facts:

1. If an orbital plane is partially eclipsed, the GLONASS satellites were often switched off for a few weeks. When the satellite signals are switched on after such a long time, a new initialization of the orbit determination process is required. Currently, such re-initializations force human interaction in the otherwise highly automated processing scheme.

2. During the maintenance phase a GPS satellite is flagged as unhealthy, but it continues to emit signals; as mentioned, we use such data at CODE for precise orbit determination. GLONASS satellites, however, do not transmit signals for an interval between 1 and 3 days at irregular intervals. The duration and frequency of these events are comparable to the maintenance periods for GPS satellites. These GLONASS maintenance events are usually unannounced. Whereas GPS maintenance periods are often associated with repositioning events, we did not notice any repositioning events for GLONASS. It is thus possible to predict GLONASS orbits over long time intervals for the re-initialization of the orbit determination process, when the satellite is again tracked by the receivers in the IGS network-even if broadcast information is not yet available.

These system specific outages are summarized in Fig. 5: If the CODE final solution contained a GLONASS satellite orbit with the usual accuracy, the day is marked by a blue square. Red squares mark days where orbit determination was of poor quality due to a limited number of receivers tracking the satellite. White squares mark days where no orbit 


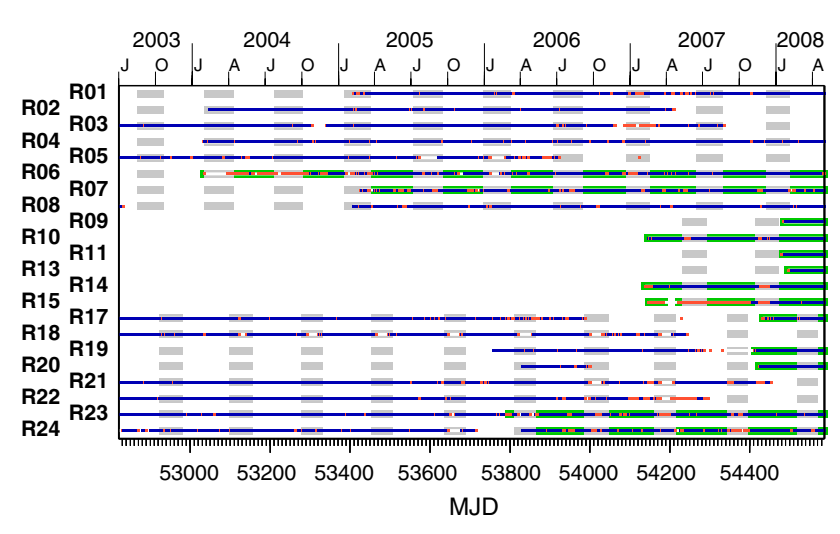

Fig. 5 Days for which orbits of the individual GLONASS satellites are provided by CODE since July 2003 are indicated by blue squares. If the orbit determination was not very reliable because of the lack of tracking data a red square is used instead (in most cases the satellites are flagged as unusable in that time). Green bars indicate intervals where the PRN slot was occupied by a new GLONASS-M satellite. Grey bars indicate eclipsing periods for the satellites at a particular orbital plane

determination was possible, because of missing data (e.g., due to inactive satellites). Many gaps (white squares) occur during the eclipsing phases marked by grey bars. Satellites R05, R18, and R21 illustrate the behavior.

In 2003, the first GLONASS-M satellite- a new generation of GLONASS satellites-was launched (R06 was running in a testing mode over several months in 2004). The replacement of an old-style satellite by a GLONASS-M satellite is indicated by green bars in Fig. 5. The current constellation mainly consists of GLONASS-M satellites, because many of the older satellites have been decommissioned. R01, R04, and R08 are the only active old generation satellites. The new satellites continue operating during eclipse phases, which is a big advantage for orbit determination. Also the lifetime of the new generation satellites seems to be longer than for the old ones. The short lifetime of old generation GLONASS satellites is another factor for the bigger variability in the GLONASS satellites constellation displayed in Fig. 4.

Let us attempt to asses the precision of the GLONASS (and GPS) orbits. For this purpose we use the ephemerides of our final orbit series of three consecutive days. The positions from the daily (independent) solutions, at 15-min intervals, are used as pseudo-observations in an orbit determination process, where only six initial osculating elements and nine empirical parameters (three constant and six once-per revolution parameters in D-, Y-, and X-directions) were determined. The RMS error of one satellite coordinate (hereafter simply referred to as RMS) is used as a precision indicator.

We do not want to include problems of marginally observed satellites and therefore display the median of the RMS over all GLONASS satellites for each day (shown with blue dots in Fig. 6). For reference the corresponding values for

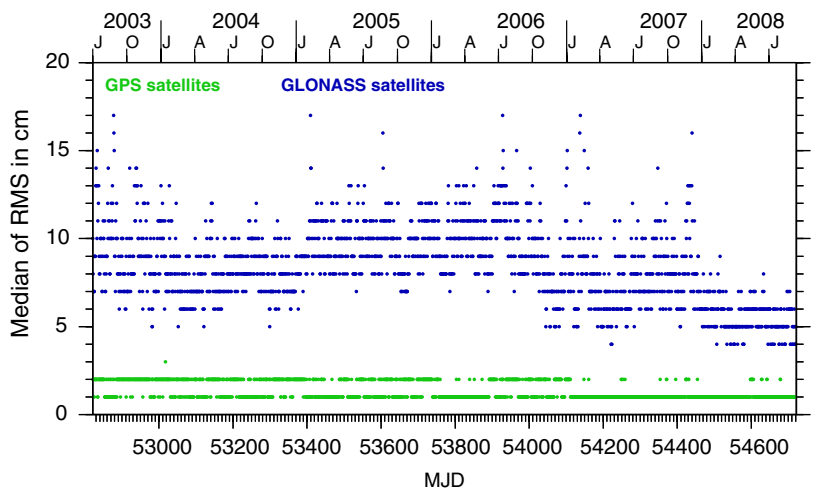

Fig. 6 Median of the RMS for the fit of a 3-day arc through the daily independent orbit solutions for the GPS (green) and GLONASS (blue) satellites obtained in the combined GPS/GLONASS processing at CODE since 2003

the GPS satellites are given in green. There is a clear correlation of the RMS with the number of stations tracking GLONASS satellites (see Fig. 1): For a long time interval the median of the RMS for the GLONASS satellites was of the order of $8-10 \mathrm{~cm}$. With the significantly increased number of GLONASS tracking stations in the IGS network this value was recently reduced to about $5 \mathrm{~cm}$. Note that the median of the RMS error is much larger than the corresponding value for the GPS satellites. This mainly reflects the smaller number of tracking stations and the less than optimal global distribution (compared to GPS). It is, however, remarkable that a long time series of GLONASS ephemerides with sub-decimeter precision are now available. This precision is sufficient for many purposes of "everyday surveys".

\subsection{Handling of GPS repositioning events}

Because GPS satellites are in deep 2:1 resonance with Earth rotation, i.e., the revolution period of GPS satellites is precisely half a sidereal day, the satellites within the same orbital plane have to be frequently repositioned to maintain a more or less regular satellite constellation. A repositioning is performed by a short thrust in along-track direction. Such a thrust may be approximated by a pulse, an instantaneous velocity change $\Delta V$ in the along-track direction. Since January 2004, CODE estimates the epochs and the sizes $\Delta V$ of these pulses using the data from the IGS network (Hugentobler et al. 2006).

The following procedure is applied: Already during the station-specific synchronization of the receiver clocks to GPS system time using the GPS code measurements, the measurement values after the pulse epoch are identified as outliers. Using this information as the starting point, the correct epoch of the pulse is obtained iteratively. Two independent arcs (separated by the pulse epoch) are introduced for the processing of the data of the corresponding satellite. The parameters 


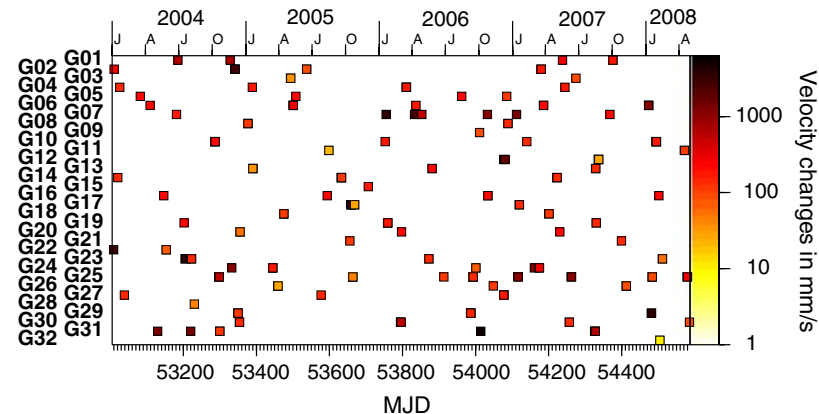

Fig. 7 Repositioning events of the GPS satellites since January 2004 as they are derived by CODE

for both orbital arcs are estimated in several iteration steps. The epoch, where both arcs have the smallest distance, is assumed to be the pulse epoch. This epoch is essential for the successful estimation of the repositioning event since it decides to which of the two arcs an observation contributes. The size, $\Delta V$, is simply the velocity difference at the pulse epoch as computed from the two arcs. Figure 7 shows the detected repositioning events since January 2004. Most of them have a a size of about $200 \mathrm{~mm} / \mathrm{s}$.

A user of the CODE orbits can independently introduce the orbit information before and after the event. In this way, the time where a satellite cannot be used during a repositioning event is minimized. Only few epochs during "very heavy" repositioning events must be excluded, because the thrust lasts for a certain amount of time.

No repositioning events were detected for the GLONASS satellites even if a satellite was inactive for several months. This is only possible because the GLONASS satellites are not in deep, but only shallow 2:1 resonance with Earth rotation (17 revolutions within 8 sidereal days; for comparison a GPS satellite carries out 16 revolutions within 8 sidereal days).

\section{Comparing GPS-only and multi-GNSS solutions}

\subsection{GPS and GLONASS orbit characteristics}

The sub-satellite track of one particular GPS satellite is repeated every day. It is therefore possible to show all subsatellite tracks for the entire GPS constellation using 1 day as an example. As long as the satellites are not moved to a different position within the orbital plane, the same ground tracks result for each day. Figure 8a shows the ground tracks for all GPS satellites during 10 days in February 2008. The GPS-specific ground tracks imply that a particular satellite follows the same azimuth-elevation paths (at maximum two visibility intervals per day) for one and the same site. This implies in particular that the observation scenarios of particular GPS satellites are-for a given latitude-

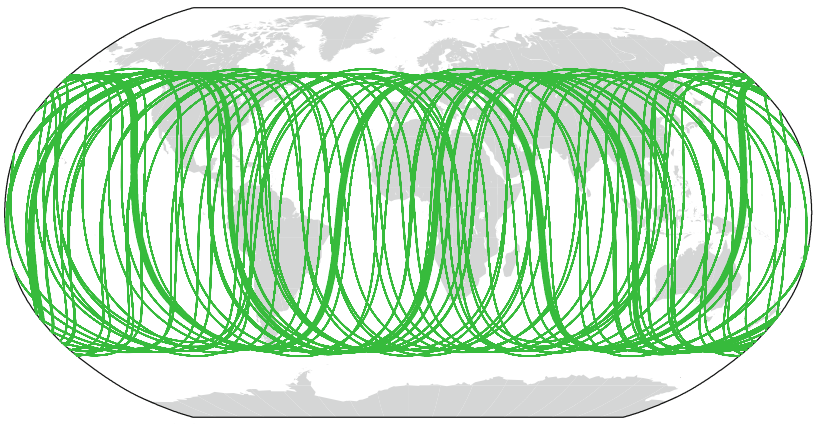

(a)

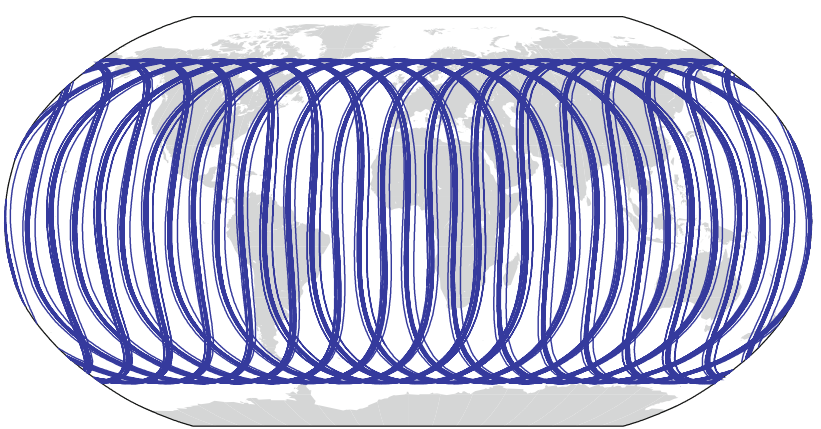

(b)

Fig. 8 Ground track of the GPS and GLONASS constellation during 10 days (day 60-69 of year 2008) in February 2008. a Ground track of the GPS satellites. b Ground track of the GLONASS satellites

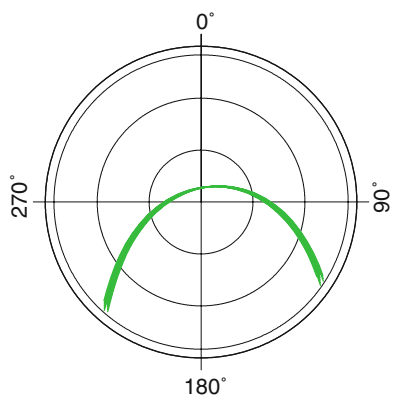

(a)

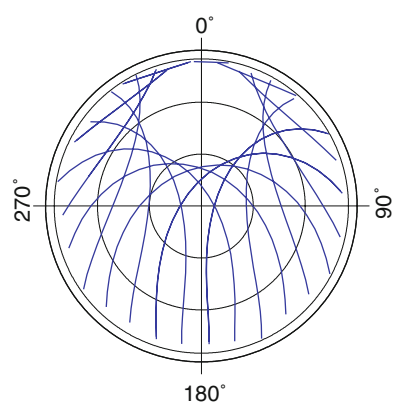

(b)
Fig. 9 Elevation-azimuth-diagram for one GPS (left) and one GLONASS (right) satellite at the location of Zimmerwald, Switzerland, accumulated during 10 days (day 60-69 of year 2008) in February 2008. a GPS satellite G06 b GLONASS satellite R06

longitude-dependent. As the IGS network is not really global and homogeneous, this fact implies that different GPS satellites are most likely not observed with the same "intensity" and with the same quality. Figure 9a shows an example for the site Zimmerwald at a Northern latitude of about $45^{\circ}$. Note that the ground track actually corresponds to 10 days, which proves that the particular GPS satellite follows the same track day after day. Only one GPS track, culminating almost at $90^{\circ}$ elevation results in this case. A site situated at the same latitude as Zimmerwald, but separated in longitude by $\pm 90^{\circ}$ would observe two tracks of the same GPS satellite 
per day, culminating at lower elevations, one in the East and one in the West.

GLONASS ground tracks are repeated after 8 sidereal days (which corresponds to a deep 17:8 resonance with Earth rotation). The ground tracks of all 16 GLONASS satellites active on the same days in 2008 are shown in Fig. 8b. The ground track of a particular satellite is shifted by $45^{\circ}$ in longitude per day. As the satellites in one and the same orbital plane are separated by $45^{\circ}$ in the full nominal constellation, the ground track generated by one particular satellite on day $i$ is the same as the ground track of its two neighbors on days $i \pm 1$. Therefore, one orbital plane of the GLONASS, in essence, generates one ground track, where all ground tracks are much steeper than the GPS ground tracks as a consequence of the 8 sidereal day repeat cycle. From the scientific perspective it is unfortunate that the arguments of latitude of the satellites in the three orbital planes are defined in such a way that the satellites in the three orbital planes all generate one and the same ground track. This characteristic may be attractive for the system operators (it reduces the number of necessary control stations) but it would be better from the scientific point of view to have a less regular pattern.

Be this as it may: It is an important difference of the GLONASS with respect to the GPS constellation that, in the average over 8 sidereal days, all sites at one and the same latitude observe each GLONASS satellite in essence in the same way (shifted only by a time offset governed by the longitude difference). Figure $9 b$, which was generated in the same way as Fig. 9a covering the time interval of 10 days, illustrates this behavior. One GLONASS satellite in essence fills the entire azimuth-elevation plot (except for the hole in the North, caused by the satellites' inclination). Due to the special selection of the arguments of latitude in the three orbital planes, Fig. $9 \mathrm{~b}$ also characterizes the ground tracks of all GLONASS satellites. As a matter of fact this leads to an 8-h repeat cycle in the satellite geometry for the stations.

As each GLONASS satellite transmits its signal on an individual frequency the impact of frequency-dependent effects such as multipath on station-specific parameters (such as coordinates and troposphere) should be reduced for this constellation. For such issues we expect a period of four sidereal days (as opposed to one sidereal day for the GPS), because GLONASS satellites separated by $180^{\circ}$ in the orbital plane use the same frequencies.

\subsection{Benefit of the combined GNSS products on navigation and rapid positioning}

The 31 GPS satellites and 16 GLONASS were active in the first quarter of the year 2008. A combined GPS/GLONASS receiver thus tracks on average $50 \%$ more satellites than a GPS-only receiver. Figure 10a shows these numbers for the

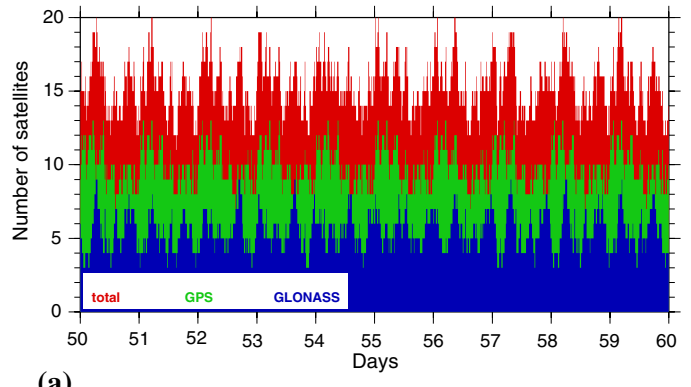

(a)

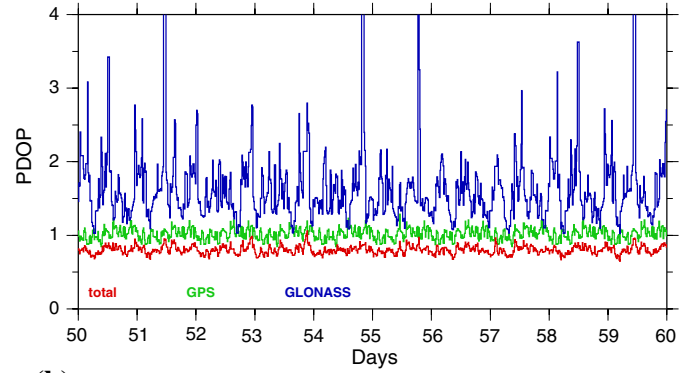

(b)

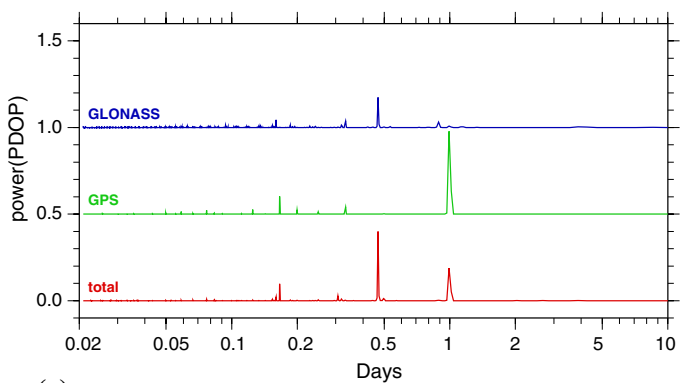

(c)

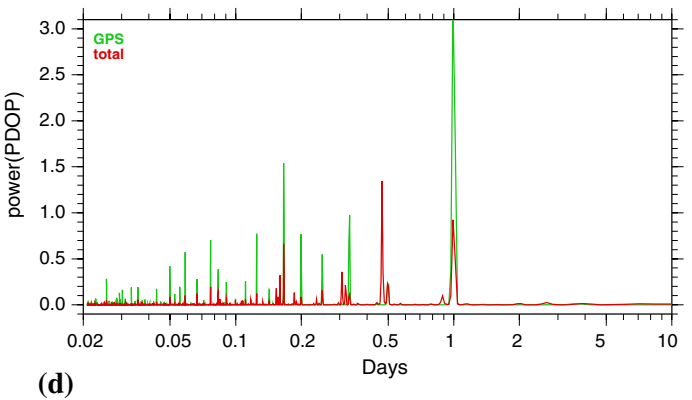

Fig. 10 Comparison of the satellite geometry for IGS station Zimmerwald for the GPS (green), GLONASS (blue), and combined (red) satellite constellation. An elevation cut-off of $5^{\circ}$ is assumed. a Number of satellites for station Zimmerwald for 10 days in 2008. b PDOP for station Zimmerwald for 10 days in 2008. $\mathbf{c}$ Normalized power spectra of the PDOP time series for station Zimmerwald for 2 months (day 20-79 of year 2008). The three curves are shifted by 0.5 for plotting d Power spectra of the PDOP time series for station Zimmerwald for 2 months (day 20-79 of year 2008). The curve for the GLONASS-only PDOP values was not plotted because it is much more noisier than the other ones because of the incomplete GLONASS constellation

Zimmerwald site, where one can see that, on average, about 15 GNSS satellites may be observed simultaneously, as opposed to 10 GPS satellites and five GLONASS satellites, individually. As the current GLONASS constellation consists 
of only 16 out of the 24 satellites of the full constellation, there are short periods where only three or fewer GLONASS satellites are in view. Nevertheless, we may expect an accuracy gain of the combined system for navigation and for positioning using short (few minutes) time spans of about $\sqrt{31 / 16} \approx \sqrt{1.5} \approx 1.22=122 \%$ in a least square adjustment. This expectation is confirmed by Fig. 10b showing the PDOP values for the GPS, GLONASS, and the multi-GNSS constellation. The PDOP value in essence gives the average of the mean errors in the three orthogonal directions North, East, Up of a position determination assuming code observations of the accuracy of one meter (remember that smaller PDOP values correspond to better satellite geometry). The same PDOP may be used for phase observations with resolved all ambiguities, where the unit would be $\mathrm{mm}$. The expected accuracy gain is not dramatic. With the full 24 satellite constellation the gain will be $\sqrt{55 / 32} \approx 1.31=131 \%$. More important, but more difficult to illustrate, is the gain in robustness of the solution.

The normalized power spectra of the GPS, the GLONASS, and the combined PDOP series in Fig. 10c differ considerably: The main feature of the PDOP spectrum for GPS is the signal at $23^{h} 56^{m}$, corresponding to one sidereal day and to the repetition of the satellite geometry at a ground station after two revolutions of the GPS satellites. The main feature of the GLONASS PDOP spectrum is the signal at $11^{h} 16^{m}$, corresponding to the GLONASS revolution period. As expected, the power spectrum of the GNSS signal contains both prominent spectral lines. At least with the current incomplete GLONASS constellation the spectral line at $11^{h} 16^{m}$ is stronger than that at $23^{h} 56^{m}$. Regarding the big reduction of the amplitude at $23^{h} 56^{m}$ due to adding the GLONASS measurements to the GPS observations-as shown in the non-normalized power spectra of Fig. 10d-this additional spectral line in the PDOP series of the combined GPS/ GLONASS processing (red line) with respect to a GPS-only series (green line) seems to be acceptable.

The difference of the PDOP spectra may have an important consequence when studying the coordinate repeatability based on daily coordinate sets (solar day) using time spans of 1-2 weeks: In case of a GPS-only solution, the satellite constellation in repeated one-by-one in every daily solution. This may lead to too optimistic results, e.g., in case of a satellite-dependent error source. Because of the higher variation in the satellite geometry within a daily solution, the repeatability of daily coordinate solution in a GLONASSonly or a combined GPS/GLONASS processing may therefore slightly worse than in a GPS-only analysis but it gives a more realistic measure of the accuracy.

From the deliberations in this subsection we may conclude that the user of the combined products has the following advantages when processing data gathered in time spans of several minutes:
- With more satellites, the solutions are much more robust, at least in the sense of a better redundancy. Outlier detection and bias identification and removal (in particular cycle slip identification and correction) are much easier. Also the success rate of ambiguity resolution within short time intervals is expected to be improved.

- The accuracy of results (point positioning, differential positioning) should be improved by the statistical $\sqrt{n}$-law, where $n$ is the number of observations. As the number of observations is proportional to the number of satellites, we expect the results to follow the same law, where $n$ is the number of simultaneously observed satellites.

In order to check the second expectation, the following test was performed: The European network solution, the CODE contribution to the EPN (European Permanent Network, Bruyninx and Roosbeek 2007), was processed in daily batches, for a 2 month interval. The orbits and the coordinates of the reference stations were introduced from the official CODE contribution to the IGS (final solution) respective to the EPN. The coordinates of the other sites and the troposphere parameters were adjusted in the experiment.

The combined GPS/GLONASS receiver at Zimmerwald observatory (ZIM2) was considered as "mildly kinematic", i.e., coordinates were estimated at 3-min intervals whereas, the ambiguities were re-introduced as known from the standard network processing. Only the GPS observations were used for all stations in the solution in the first part of the test. All observations (GPS and GLONASS) were used in the second part of the test. It would have been most thorough to generate a third solution using only the GLONASS measurements. However, in view of the limited number of simultaneously visible GLONASS satellites (at times there are only three satellites, see Fig. 10a), such a solution makes little sense.

The obtained time series of kinematic positions for Zimmerwald station with a sampling of $3 \mathrm{~min}$ (all in all 30,240 data points within 63 days) was analyzed in two different ways: At first the Allan deviation (see Allan 1987) is generated to access the impact of the additionally used GLONASS measurements on the obtained "kinematic trajectory". In a second analysis the time series was devided into subintervals to compute the standard deviation for the mean value of the interval. The length of these subintervals was varied to study the influence of the additional observations on a fictive coordinate solution obtained from the different intervals of measurements.

In Figure 11 the Allan deviations is displayed generated with the two sets of 3 min solutions for Zimmerwald station. The Allan deviations referring to a spacing of $\tau$ between data points are given by 


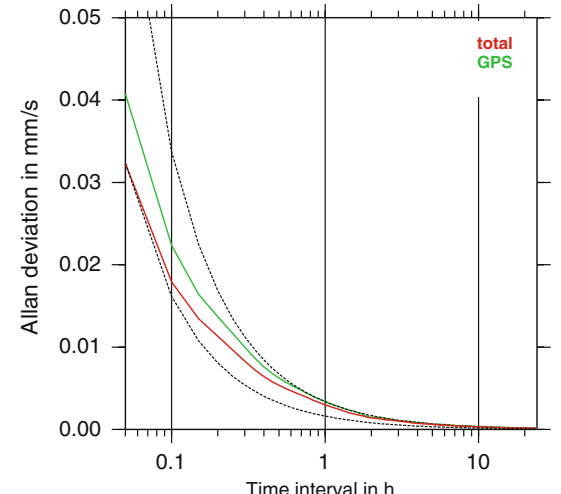

(a)

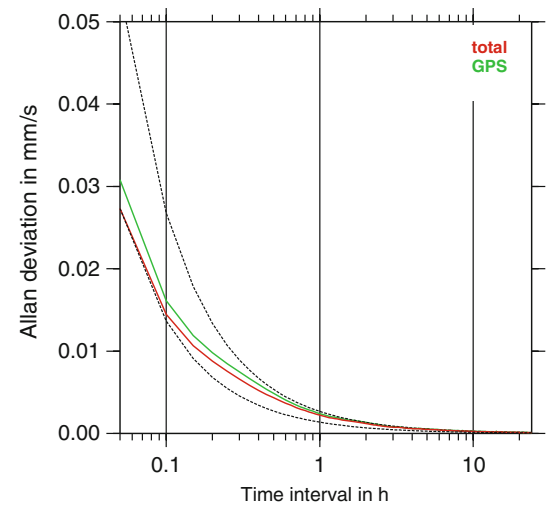

(b)

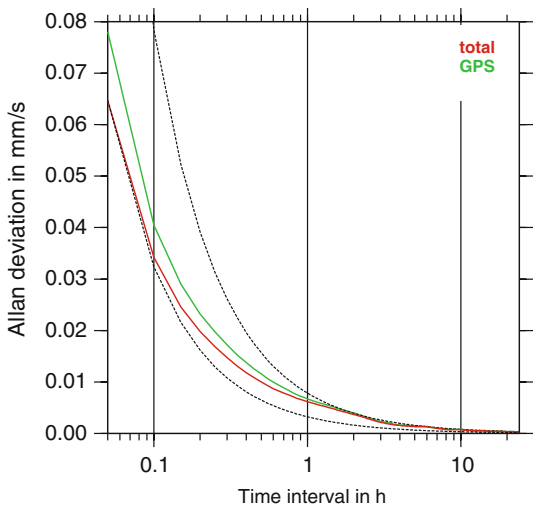

(c)
Fig. 11 Allan deviations of the kinematic positions (at 3-min intervals over 60 days) of the combined GPS/GLONASS receiver in Zimmerwald (ZIM2) using only GPS measurements (green line) and observations from both GNSS (red line), respectively. The unusual non-logarithmic scale was used to show the differences between the two curves more clearly. The dotted lines indicate the slope of -1 for a white noise behaviour. a North component b East component $\mathbf{c}$ Up component

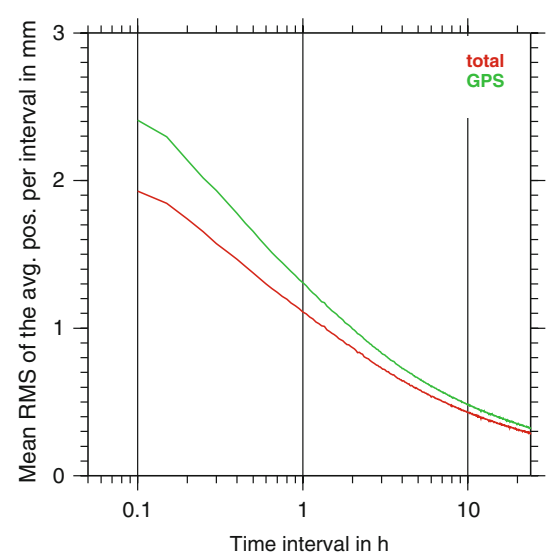

(a)

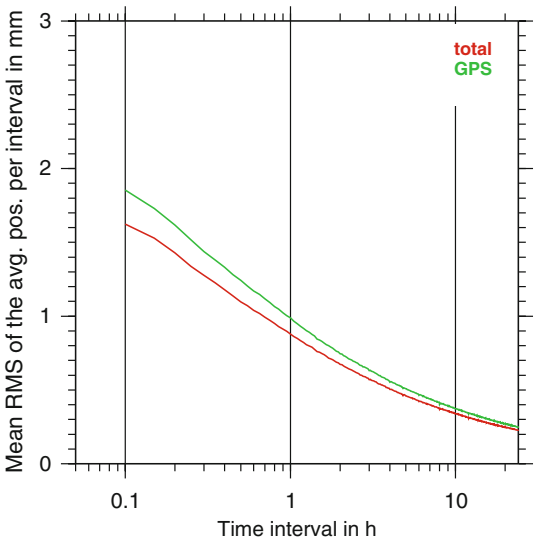

(b)

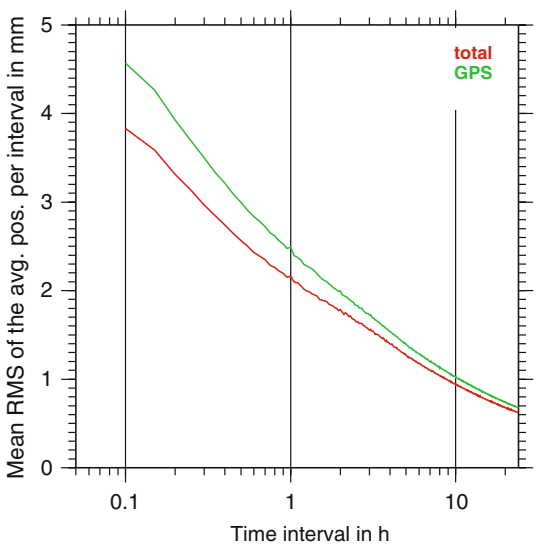

(c)
Fig. 12 Standard deviation of the mean position computed over a certain time interval-derived from the kinematic positions (at 3-min intervals over 60 days) of the combined GPS/GLONASS receiver in
Zimmerwald (ZIM2) using only GPS measurements (green line) and observations from both GNSS (red line), respectively a North component b East component $\mathbf{c}$ Up component
$\sigma(\tau)=\sqrt{\frac{1}{2(N-2) \tau^{2}} \cdot \sum_{i=1}^{N-2}\left(x_{i}-2 x_{i+1}+x_{i+2}\right)^{2}}$,

where the data values $x_{k}, k=i, i+1, i+2$ refer to epochs separated by $\tau$.

The red line in Fig. 11 refers to the combined processing of GPS and GLONASS measurements, whereas the green line is obtained from the GPS-only solution. For short time intervals (up to a few minutes) the Allan deviation is dominated by the noise of the carrier phase (see also Dach et al. 2007a). In this domain the additional GLONASS measurements help according to the $\sqrt{n}$-law to reduce the noise of the kinematic positions by $20-25 \%$. For longer intervals - let us say half an hour or more - the improvement becomes very small. For intervals of $1 \mathrm{~h}$ and longer the difference of both curves is even smaller. It means that the additional GLONASS measurements help to improve mainly the epoch-to-epoch stability of the obtained kinematic trajectory.

An alternative analysis of the 3-min series of the kinematic positions for the Zimmerwald station is presented in Fig. 12. The time series has been divided into intervals, each with $N$ 3-min epochs. The positions estimated from all the observations within an interval may be approximated by forming an arithmetic mean from the 3-min kinematic positions of this interval. Than the quality of such a mean interval positions can assessed from the standard deviation of the mean computed by

$$
\sigma=\sqrt{\frac{1}{N \times(N-1)} \cdot \sum_{i=1}^{N}\left(x_{i}-\bar{x}\right)^{2}} \text { with } \bar{x}=\frac{1}{N} \cdot \sum_{i=1}^{N} x_{i} .
$$


The length of the interval to compute the fictive mean coordinate has been varied from a few epochs over several hours up to 1 day.

The green line in Fig. 12 represents the results derived from the GPS-only solution whereas the red line refers to the combined GPS/GLONASS solution. The benefit for the short intervals (e.g., $6 \mathrm{~min}$ or $0.1 \mathrm{~h}$ ) is again $20-25 \%$-as it was found in the Allan deviation. For an hourly processing the analysis still promises a benefit of $5-10 \%$ due to the additionally processed measurements-for longer interval lengths the benefit decreases rapidly, but the red curve (GPS/GLONASS solution) remains slightly below the green line (GPS-only solution).

The decreasing benefit due to adding the GLONASS to the GPS observations in the kinematic solution for time intervals longer than $1 \mathrm{~h}$ may need a further discussion: Beside the $\sqrt{n}$-law, one should also not forget the general modelling aspects in the GNSS processing (e.g., troposphere) acting in the same (or at least very similar) way on the measurements of each GNSS. Concerning GLONASS in this particular case it must stated, that less ambiguities ${ }^{1}$ are resolved than for GPS. A degradation of the benefit from the GLONASS measurements for the combined solution is expected according to the experience with ambiguity resolutions in the GPS-only environment. On the other hand, even an ambiguity resolution rate of $85-95 \%$ in the Swiss national network (see Sect. 4) gives very similar Allan deviation plots when the stations are processed in a kinematic mode. This might indicate that beside the ambiguity resolution there seem to be other effects that are not adequately modelled in the processing (e.g., the antenna phase center models for GLONASS receiver and satellite antennas).

\subsection{Impact of GLONASS on the CODE global products}

Based on the findings of the previous paragraph we cannot expect major improvements by adding the GLONASS measurements to the GPS data in the coordinate series estimated in the CODE routine processing-the coordinates are only available in daily batches.

Figure 13 shows the daily RMS values in North, East and Up components of the coordinate differences between two solutions with and without using the GLONASS observations in the processing. About 150 sites are included in the

\footnotetext{
${ }^{1}$ In the CODE IGS-processing the GLONASS ambiguity resolution is only enabled for baselines shorter than $20 \mathrm{~km}$ since August 2007 (success-rate is close to $100 \%$ ) whereas for the European solution the GLONASS ambiguities is only allowed between satellites using the same frequencies for test purposes when using the QIF-ambiguity strategy for baselines up to 2,000 km length (success-rate is about $30 \%$ with respect to all ambiguities). A detailed description of the ambiguity resolution strategies in the Bernese GPS Software is given in Mervart (1995).
}

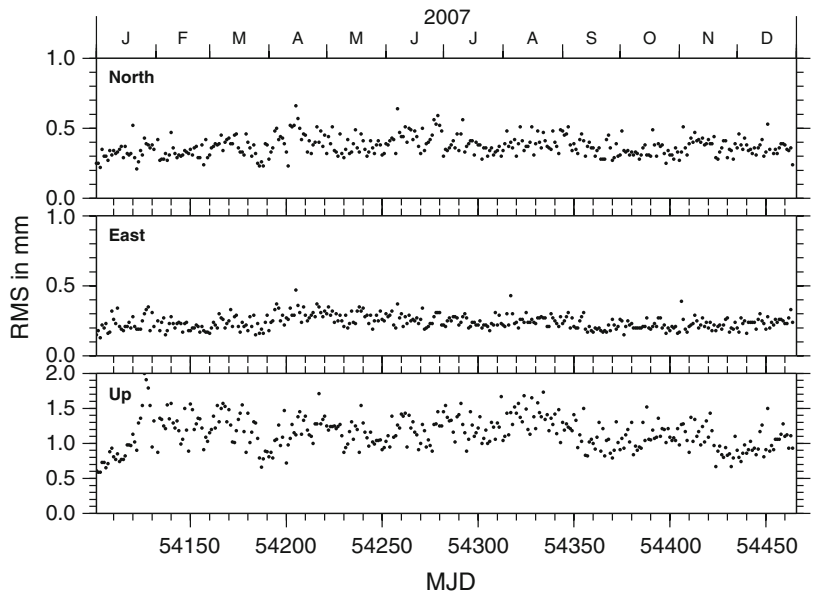

Fig. 13 RMS of the coordinate differences obtained in daily GPS-only respective combined GPS/GLONASS solutions in a global network during the year 2007

global analysis corresponding very closely to the CODE IGS analysis (i.e., solving for all relevant parameters, including orbit, coordinates, troposphere, and ambiguities that are not resolved to their integer values). Figure 13 has a simple message: The global reference frame is only marginally changed when adding or leaving out GLONASS measurements in the processing.

The comparison of the GPS orbits in a GPS-only and a in a combined GPS/GLONASS processing shows differences in the order of $\approx 1 \mathrm{~cm}$ (up to $2 \mathrm{~cm}$ for some cases). These are small values, which correspond to the mm-differences in the station coordinates. We conclude that the addition of GLONASS into the analysis currently has no significant impact on the GPS orbits, i.e., including GLONASS does not help nor hurt the determination of global parameters from GPS data.

The same conclusion can be drawn for the Earth rotation parameters. The differences in the results with and without using GLONASS data are insignificant.

\section{GNSS applications using the Swiss AGNES network}

The Automated GNSS Network for Switzerland (AGNES) and the Swiss Positioning Service (swipos) constitute an important part of the geodetic infrastructure of Switzerland. Since AGNES is a multifunctional reference network not only for applications in national surveying but also for scientific studies and positioning services, swisstopo had to find a compromise between the continuity of the observations and the rapid alignment to the demands and developments of the market: All major manufacturers of GNSS receivers have been designing combined receivers for both, GPS and GLONASS, since 2006. The greater number of satellites does 
bring about improvements in the positioning service swipos because the availability and performance of the service has increased in difficult terrain (built up areas, narrow valleys, etc.). In order to keep up with this development, swisstopo adapted its network AGNES, consisting of 31 permanently operating stations, to the new technical demands.

During summer 2007 the first 11 AGNES stations was converted from GPS-only to new combined GPS/GLONASS receivers. Until April 2008 nearly all AGNES sites have been equipped with the new receivers. The present status of the network is given in Fig. 14-red circles indicate the location of the combined GPS/GLONASS receivers. To assure continuity, the old GPS-only receivers are planned to be operated simultaneously with the new GPS/GLONASS receivers on ten AGNES stations (Brockmann et al. 2007; Ineichen et al. 2007). Six of these double-sites are currently (April 2008) installed (yellow circles in Fig. 14).

The monitoring of the stability of site coordinates is an important part of AGNES to guarantee reference frame maintenance. To fulfill this demand, the complete AGNES network is analyzed with about 40 other EPN/IGS sites on an hourly and daily basis using the Bernese GPS Software, Version 5.0. The ultra-rapid and the final orbit products from the CODE analysis center of the IGS are used to process these stations, because combined multi-system products are not available through the IGS.

To guarantee highest precision, all of the new GNSS antennas were first calibrated by the company Geo++ in Germany, which is specialized to perform such calibrations, e.g., Wübbena et al. (2006). Absolute elevation- and azimuthdependent antenna phase center variations were derived for GPS (individual corrections for each antenna) and for

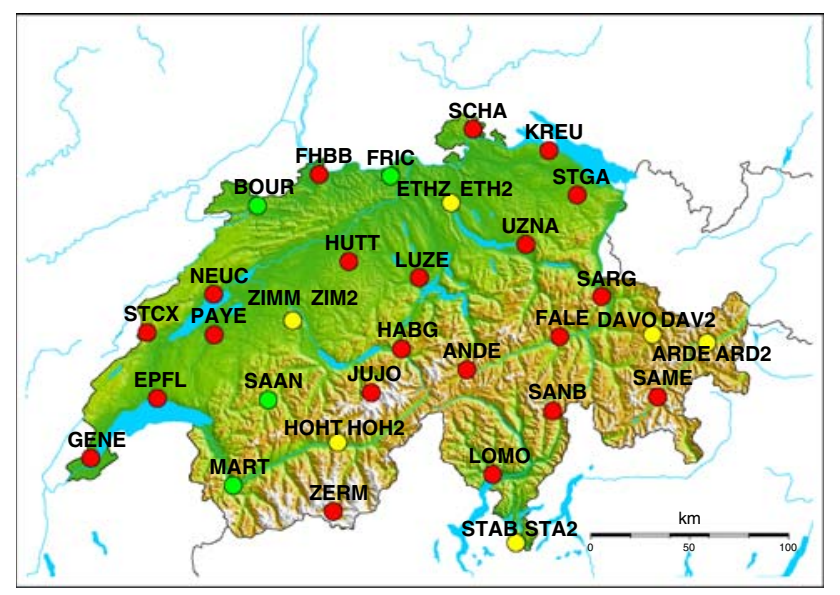

Fig. 14 Status of the AGNES network (April 2008): red circles indicate new GPS/GLONASS receivers, green circles show the locations of stations with a GPS-only tracking receivers, yellow circles represent stations where a GPS-only and a GPS/GLONASS receiver are operating simultaneously

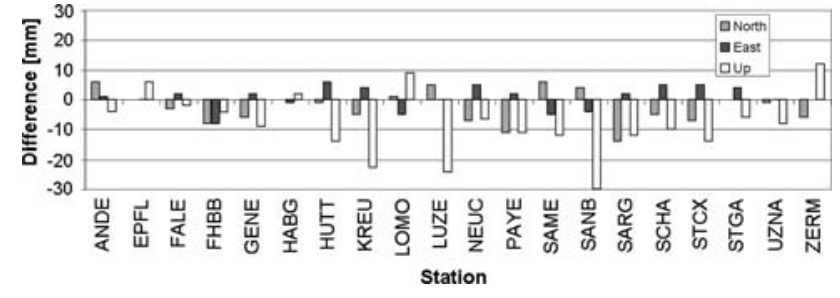

Fig. 15 Discontinuities in the coordinate time series due to the replacement of the antennas at 20 AGNES sites

GLONASS (one set of group corrections). Discontinuities in the coordinate time series, which occurred due to the change of the antenna, were below several millimeters for the horizontal direction and at maximum $3 \mathrm{~cm}$ for the vertical direction (see Fig. 15). Another consequence of the interruption is the reduced accuracy of the velocity estimation. An analogue process is currently ongoing within the IGS network (see Fig. 1). It may be expected in a much shorter interval when the Galileo system and the corresponding hardware is in place.

Ambiguity resolution of GPS/GLONASS combined observations was not possible using Bernese GPS Software beginning of 2007 (Schaer 2007). In close cooperation with the University of Bern, swisstopo developed several improvements (optimization of the generation of short GNSS-baselines, improved preprocessing, improved GNSS ambiguity resolution). With these modifications it became possible to successfully implement ambiguity resolution since June 2007 for the post-processing solutions and since September 2007 for the near real-time processing. No double-difference ambiguities in between GPS and GLONASS are solved for. Also, the QIF ambiguity resolution strategy (Mervart 1995) only allows it to solve ambiguities referring to the same frequencies. For all baselines between Swiss stations (on average separated by $50 \mathrm{~km}$ ) and for all GNSS-baselines shorter than $200 \mathrm{~km}$, the combined widelane and narrowlane ambiguity resolution strategy enables a successful ambiguity resolution of $85-95 \%$, which is comparable to the success rate when analyzing GPS data, only.

Similar to the findings in Sect. 3.2, the results presented in Ineichen et al. (2008) for station-depending parameters (coordinates and troposphere) are also very comparable. This is true for daily results as well as for results derived from a near real-time processing of the data in hourly sliding batches of 8-h. These solutions are finished and submitted within $30 \mathrm{~min}$. The main reason for enhancing the AGNES network with GLONASS is therefore the increasing availability of the Swiss Positioning Service (swipos) for surveyors measuring positions on centimeter level in realtime and in difficult environments-about $60 \%$ of the swipos users indicate to use both available GNSS in combination. 


\section{Summary}

Since May 2003 CODE offers in its products GPS and GLONASS ephemerides referring to one and the same reference frame [in essence a realization of the most recent ITRF release(s)]. According to the weekly reports of the IGS Analysis Coordinator, the GPS ephemerides are consistent on the $1-2 \mathrm{~cm}$ level with the IGS combined products. From the combination of the GLONASS orbits, a consistency level of $3-5 \mathrm{~cm}$ between the contributing ACs ${ }^{2}$ may be derived.

In this article we assessed the precision of the GLONASS orbits to be of the order of $8 \mathrm{~cm}$ RMS per coordinate, initially, (in 2004) and $5 \mathrm{~cm}$, today. These figures will get closer to the GPS figures (a) with the completion of the full GLONASS configuration and (b) with the increase of the number and the improvement of the global distribution of state of the art combined GPS/GLONASS receivers.

We have pointed out important differences between the GPS and the GLONASS observation geometries: The observation geometry (expressed by the PDOP) shows a strong once-per-revolution signal for GLONASS, whereas there is a prominent daily signal (sidereal day) in the GPS PDOP. The observation geometry of the combined GPS/GLONASS PDOP contains, as expected, both signals.

The power spectrum of the GPS-only PDOP shows a prominent signal at one sidereal day. This has to expected, as the entire configuration is repeated after one sidereal day. Two aspects are important in this context:

- The solar day is very close to the sidereal day (difference of about $4 \mathrm{~min}$ ). Using the solar day as sampling interval, e.g., for coordinate time series may lead to an over-estimation of GPS accuracies derived from the repeatability of the daily positions.

- The sampling of a signal over a period which is slightly different from the prominent period in the GPS PDOP spectrum (in the GPS case one sidereal day) may generate spurious beat signals-which were, as a matter of fact, described by Ray et al. (2008).

The power spectrum of the GLONASS-only PDOP in essence has one prominent spectral line corresponding to the revolution period of $11^{h} 16^{m}$ of the GLONASS satellites. There is almost no power in the daily or quasi-daily domain. If we would have the same number of satellites in both constellations we would, therefore, expect for GLONASS-only solutions to have

1. A slightly reduced (solar) daily coordinate repeatability as compared to GPS.

\footnotetext{
2 Please note that only four centers provide GLONASS orbits but only two of them contribute also to the traditional IGS products.
}

2. But a reduced quasi-annual beat signal-the current GLONASS does not allow it to study this aspect with sufficient precision.

We have shown that the statistical expectation (improvement of the results of GNSS surveys with $\sqrt{n}$, where $n$ is the number of (simultaneously available) satellites, roughly holds when analyzing GNSS observations from short (few minutes) data spans. This is an important benefit for many users of GNSS products, in particular for the surveying and (potentially) the navigation communities. For longer data spans, the advantages soon become much smaller, or even marginal. This means that (unmodelled) systematic effects in the data analysis dominate with respect to the pure noise of the observations.

Currently the contribution of GLONASS to the global products, in particular to the reference frame (set of station coordinates) and the Earth rotation parameters cannot be clearly identified. We are confident that this situation will change with the deployment of the full GLONASS constellation and the improvement of the global tracking network equipped with combined receivers.

The Swiss Permanent Network AGNES, which was enhanced from GPS to GPS/GLONASS in 2007 as one of the first networks consequently gathers and analyzes GPS and GLONASS observations and makes the combined products available to its user community. AGNES uses the CODE/IGS products (including the GLONASS orbits) for its analyses and provides a good example for the link from the GNSS activities within the IGS to daily surveying activities. It demonstrates the IGS is not just an "academic environment" to study GNSS phenomena. The IGS products are very central for the daily work of a very big user community. Their needs and wishes should be kept in mind in all scientific activities within the IGS. The CODE consortium as a joint venture between the scientific (AIUB) and application (swisstopo and BKG) part stands for both sides.

\section{References}

Allan D (1987) Time and frequency (time-domain) characterization, estimation, and prediction of precision clocks and oscillators. In: IEEE transactions of UFFC, vol UFFC-34(6)

Beutler G, Brockmann E, Gurtner W, Hugentobler U, Mervart L, Rothacher M (1994) Extended orbit modeling techniques at the CODE processing center of the International GPS Service for Geodynamics (IGS): theory and initial results. Man Geod 19:367-386

Brockmann E, Kistler M, Marti U, A Schlatter BV, Wiget A, Wild U (2007) National report of Switzerland: new developments in Swiss national geodetic surveying. In: Torres J, Hornik H (eds) Subcommission for the European reference frame (EUREF)

Bruyninx C, Roosbeek F (2007) EPN status and new developments. In: Torres J, Hornik H. (eds) Subcommission for the European Reference Frame (EUREF) 
Dach R, Beutler G, Gudmundson H (2007a) Analysis of GPS data from an antarctic ice stream. In: IUGG general assembly, Perugia, 2-13 July, 2007, IAG Symposia (in press)

Dach R, Hugentobler U, Meindl M, Fridez P (eds) (2007b) The Bernese GPS software version 5.0. Astronomical Institute, University of Bern

Fliegel HF, Gallini TE (1996) Solar force modeling of block IIR Global Positioning System satellites. J Spacecr Rockets 33(6):863-866

Hugentobler U, Meindl M, Beutler G, Bock H, Dach R, Jäggi A, Urschl C, Mervart L, Rothacher M, Schaer S, Brockmann E, Ineichen D, Wiget A, Wild U, Weber G, Habrich H, Boucher C (2006) CODE IGS analysis center technical report 2003/2004. In: Gowey K, Neilan R, Moore A (eds) IGS 2004 technical reports. Jet Propulsion Laboratory, Pasadena, IGS Central Bureau (in press)

Ineichen D, Brockmann E, Schaer S (2007) Enhancing the Swiss permanent GPS network (AGNES) for GLONASS. In: Torres J, Hornik H (eds) Subcommission for the European reference frame (EUREF)

Ineichen D, Brockmann E, Schaer S (2008) Processing combined GPS/GLONASS data at swisstopo's local analysis center. In: Ihde J, Hornik H (eds) Subcommission for the European reference frame (EUREF)

Ineichen D, Springer T, Beutler G (2003) Combined processing of the IGS and the IGEX network. J Geod 75(11):575-586
Mervart L (1995) Ambiguity resolution techniques in geodetic and geodynamic applications of the Global Positioning System. Geodätisch-geophysikalische Arbeiten in der Schweiz, Band 53. Schweizerische Geodätische Kommission, Institut für Geodäsie und Photogrammetrie, Eidg. Technische Hochschule Zürich, Zürich

Ray J, Altamimi Z, Collilieux X, van Dam T (2008) Anomalous harmonics in the spectra of GPS position estimates. GPS Sol 12(1):55-64

Schaer S (2007) Inclusion of GLONASS for EPN analysis at CODE/swisstopo. In: Torres J, Hornik H (eds) Subcommission for the European reference frame (EUREF)

Schaer S, Hugentobler U, Dach R, Meindl M, Bock H, Urschl C, Beutler G (2005) GNSS analysis at CODE. In: Meindl M (ed) Celebrating a decade of the international GPS service-workshop and symposium, 2004, Bern, March 1-15, 2004

Springer TA, Beutler G, Rothacher M (1999) A new solar radiation pressure model for the GPS satellites. GPS Sol 3(2):50-62

Wübbena G, Schmitz M, Boettcher G (2006) Absolute GNSS antenna calibration with a robot: repeatability, GLONASS, and carrierto-noise pattern. In: Tenth EUPOS®ICS, 22-24 November, Budapest, Hungary 